\title{
Function of Vascular Smooth Muscle and Its Sympathetic Innervation in the Newborn Dog*
}

\author{
D. L. Boatman, R. A. Shaffer, R. L. Dixon, † and M. J. Brody \\ (From the Department of Pharmacology, College of Medicine, State University of Iowa, \\ Iowa City, Iowa)
}

Review of the literature pertaining to circulatory control in the newborn reveals that the knowledge of cardiovascular physiology at this stage of development is both incomplete and conflicting. The status of the subject may be illustrated by examining studies on baroreceptor mechanisms in the neonate. Some investigators have indicated that these reflex adjustments are not fully operative in the newborn (1-3), whereas others believe them to be quite functional (4-7). It is important to realize that most of these investigations have been of a qualitative nature and do not imply that quantitatively control of the newborn circulation is the same as in the adult. Many of the studies thus far on the newborn circulation have been limited to blood pressure and heart rate measurements. We feel that only a minimal amount of information can be derived from studies of this nature and that investigations on separate vascular beds might be useful for a better understanding of the factors controlling newborn circulation. It was the purpose, therefore, of this investigation to examine the responses of an isolated vascular bed to sympathetic neurotransmitters and sympathetic nerve stimulation in order to evaluate the functions of vascular smooth muscle and its innervation in the newborn.

* Submitted for publication May 29, 1964 ; accepted October 22, 1964.

A preliminary report of this study was presented to the Midwest Section of the American Federation for Clinical Research, Chicago, October 1963 (Clin. Res. 1963 11, 287).

This investigation was supported in part by grants from the Iowa and American Heart Associations and by U. S. Public Health Service training grant 5T1 GM-141.

$\dagger$ Present address: Laboratory of Chemical Pharmacology, National Cancer Institute, Bethesda, Md.

\section{Methods}

Twenty-five mongrel puppies from six litters and five adult mongrel dogs were used in this study. Experiments were performed on the 25 puppies divided evenly in eight groups of 1 day and 1, 2, 4, and 8 weeks. The age groups were randomized from each litter to minimize inherent litter to litter variation. All animals were anesthetized with sodium pentobarbital administered by intravenous titration. To minimize variation in responses that might be attributed to depth of anesthesia, the amount of anesthetic administered was just sufficient to attain light surgical anesthesia. Adults received approximately 30 $\mathrm{mg}$ per $\mathrm{kg}$, and neonates usually required less. Newborn animals were prepared for perfusion of the hindquarters using a modification of the technique previously described in detail for rats by Brody, Shaffer, and Dixon (8). Adult dogs were prepared for the same procedure by the technique described by Beck (9). The trachea was cannulated and respiration maintained with a Harvard respiration pump. The external jugular vein was cannulated for intravenous injection of drugs. The abdominal aorta was exposed through a mid-line incision in the newborn and a flank incision in adults and was separated from the adherent connective tissue. After the administration of $5 \mathrm{mg}$ per $\mathrm{kg}$ heparin sodium (100 U per $\mathrm{mg}$ ), the aorta was cannulated with tubing leading to a model T6 (adult) or T8 (newborn) Sigmamotor pump. The aorta was again cannulated just before the bifurcation into the common iliacs with the tubing leading from the pump.

Perfusion pressure was monitored from a T-tube connection located between the constant flow pump and the isolated perfused hindlimbs. In all experiments perfusion pressure was adjusted to a level approximating mean systemic blood pressure. Since the pump perfused blood at a constant volume flow over a wide range of inflow and outflow pressures, changes in perfusion pressure were directly proportional to changes in peripheral vascular resistance. Systemic blood pressure was recorded from another $\mathrm{T}$-tube connection located between the pump and aorta. Tubing from both T-tube connections led to Statham arterial pressure transducers that were connected to a direct writing oscillograph. One lumbar sympathetic chain was isolated at L3-L4, cut centrally, and stimulated electrically through a small. bipolar electrode with square wave impulses from a Grass stimulator. 
Drugs utilized in this study were norepinephrine bitartrate, epinephrine hydrochloride (both expressed as free base), synthetic valine-5-angiotensin-II amide, ${ }^{1}$ atropine sulfate, and tyramine hydrochloride.

Statistical analyses were performed as described by Snedecor (10), and differences were considered to be significant when the probability level was less than 0.05 .

\section{Results}

Sympathetic nerve stimulation. As illustrated in Figure 1, electrical stimulation of a lumbar sympathetic nerve to the isolated hindquarters produces a decrease in vascular resistance, i.e., vasodilatation, in the 1-day- and 1- and 2-weekold puppies. A summary of the responses produced by nerve stimulation at the various ages is shown in Table I. There is no significant difference in the dilatations produced by nerve stimulation at these three early ages. In all experiments in which dilatation was observed it was abolished following intravenous administration of atropine sulfate, $0.2 \mathrm{mg}$ per $\mathrm{kg}$. Nerve stimulation produced an increase in peripheral vascular resistance in 4- and 8-week-old and adult dogs, and these constrictor responses increased in magnitude with age.

Epinephrine and norepinephrine. Intra-arterial

${ }^{1}$ Ciba Pharmaceutical Co., Summit, N. J. injections of epinephrine and norepinephrine in doses of 1 and $2 \mu \mathrm{g}$ produced similar increases in perfusion pressure at all ages. The effects of epinephrine $(2 \mu \mathrm{g})$ on perfusion pressure in all age groups are illustrated in Figure 1, and a summary of these data is shown in Table I. The responses produced by epinephrine and norepinephrine are significantly greater at 4 weeks of age compared to those responses seen at 1 day and in the adult ( $\mathrm{p}<0.05$ in both comparisons). The remainder of the points are not significantly different from each other. There are no differences in the responses produced by epinephrine as compared to norepinephrine.

Tyramine and angiotensin. The summarized data of the perfusion pressure responses obtained with tyramine and angiotensin are shown in Table I. The intra-arterial injection of $50 \mu \mathrm{g}$ of tyramine produced a decrease in resistance in the 1-day-old animals, whereas equivalent increments in perfusion pressure were observed in the 1 -week to adult age groups. Although there appears to be a trend for the magnitude of constrictions produced by tyramine to increase with age, this trend is not statistically significant due to the high variability of the responses. The intra-arterial injection of $0.5 \mu \mathrm{g}$ of synthetic angiotensin produced equivalent increases in perfusion

TABLE I

The effects of sympathetic nerve stimulation and vasoconstrictors on perfusion pressure in the hindquarters of newborn, young, and adult dogs*

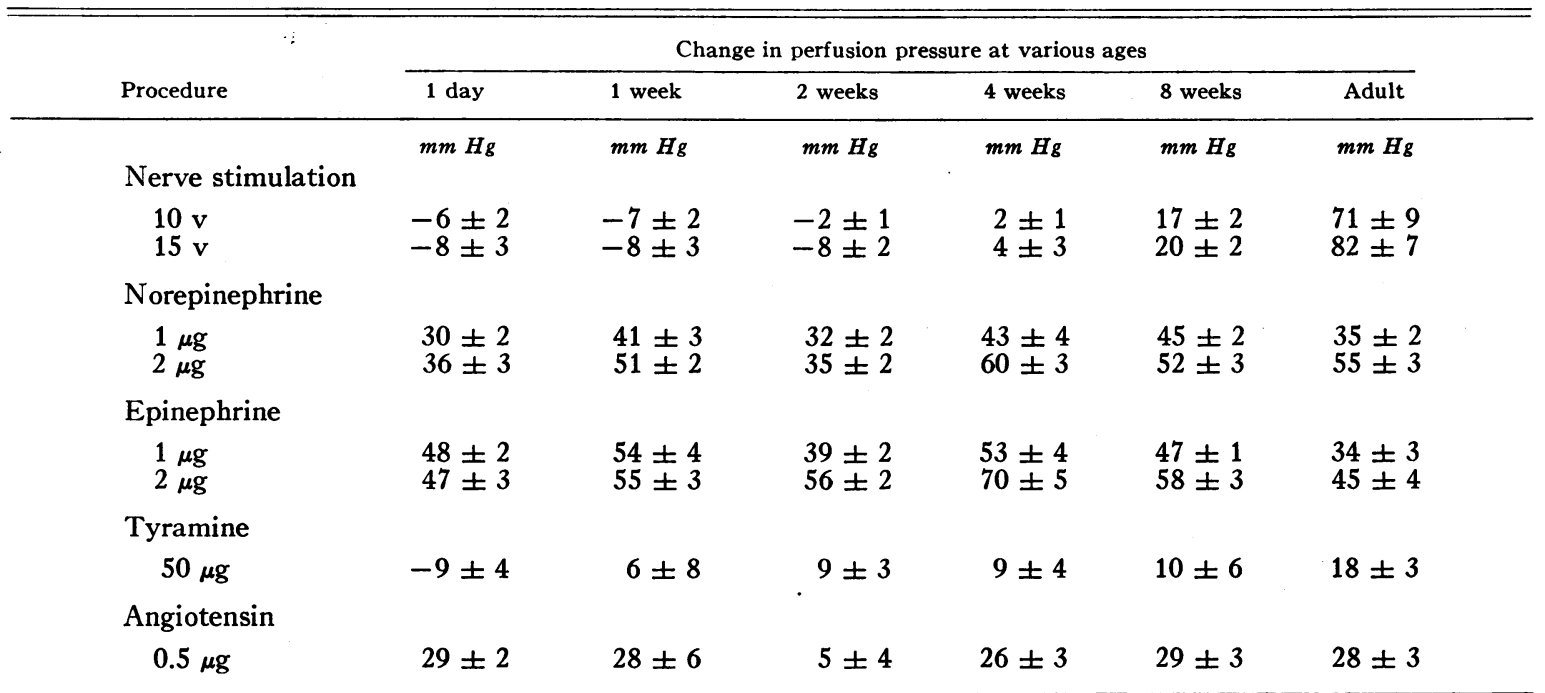

* Values represent means \pm standard errors from five animals in each group. Vasoconstrictors administered intraarterially. Nerve stimulation parameters: duration, 30 seconds; frequency, $20 \mathrm{cps}$; pulse duration, 2 msec. 

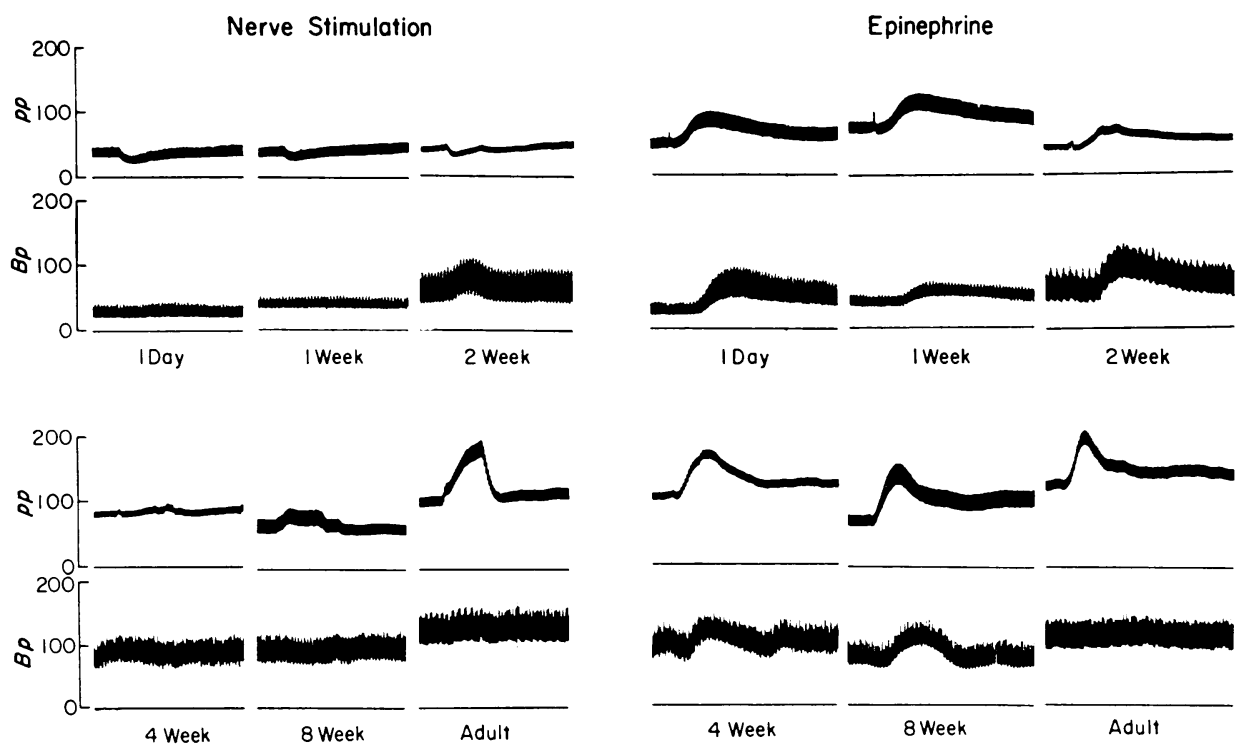

Fig. 1. Effects of nerve stimllation and epinephrine on isolated perfused hindlimbi OF THE DOG. BP, blood pressure; PP, perfusion pressure (both expressed as millimeters $\mathrm{Hg}$ ). Left panel illustrates responses to electrical stimulation of one lumbar sympathetic nerve trunk in 1-day-, 1-, 2-, 4-, and 8-week-old, and adult dogs. Nerve stimulation parameters are $10 \mathrm{v}$, $20 \mathrm{cps}, 0.2 \mathrm{msec}, 30$ seconds duration. Epinephrine (right panel) was administered intraarterially $(2 \mu \mathrm{g})$.

pressure in all groups except that at 2 weeks of age, when the increase in perfusion pressure was significantly less $(\mathrm{p}<0.01)$.

Duration of iasoconstrictor responses. From the experiments using epinephrine, norepinephrine, and angiotensin, the time required for the increases in perfusion pressure to return to onehalf of their peak values (half-lives) was calculated. The results of this analysis are summarized in Table II. The duration of the re- sponses for epinephrine and norepinephrine in animals younger than 4 weeks is significantly longer $(p<0.01)$ than those of the adult animals. At 4 weeks and older the duration of these responses is not significantly different from those seen in the adult. The appearance of rasoconstriction induced by nerve stimulation (4 weeks) occurs at the same time when the duration of constrictor responses produced by the catecholamines becomes equivalent to the adult.

TABLE II

Durations of constrictor responses in the perfused hindquarters of newborn, young, and adult dogs*

\begin{tabular}{|c|c|c|c|c|c|c|}
\hline \multirow[b]{2}{*}{ Procedure } & \multicolumn{6}{|c|}{ Half-lives of constrictor responses at various ages } \\
\hline & 1 day & 1 week & 2 weeks & 4 weeks & 8 weeks & Adult \\
\hline \multicolumn{7}{|c|}{ Epinephrine } \\
\hline $1 \mu \mathrm{g}$ & $133 \pm 10$ & $73 \pm 8$ & $58 \pm 3$ & $48 \pm 2$ & $45 \pm 3$ & $32 \pm 2$ \\
\hline \multicolumn{7}{|c|}{ Norepinephrine } \\
\hline $1 \mu \mathrm{g}$ & $105 \pm 11$ & $73 \pm 3$ & $73 \pm 1$ & $52 \pm 2$ & $52 \pm 2$ & $49 \pm 2$ \\
\hline \multicolumn{7}{|c|}{ Angiotensin } \\
\hline $0.5 \mu \mathrm{g}$ & $116 \pm 8$ & $95 \pm 4$ & $70 \pm 2$ & $67 \pm 1$ & $76 \pm 5$ & $84 \pm 3$ \\
\hline
\end{tabular}

* Values represent means \pm standard errors from five animals in each group. All agents administered intraarterially.

$\dagger$ Represents time required for constrictor response to return to $50 \sigma_{i}^{-}$of perfusion pressure change. 


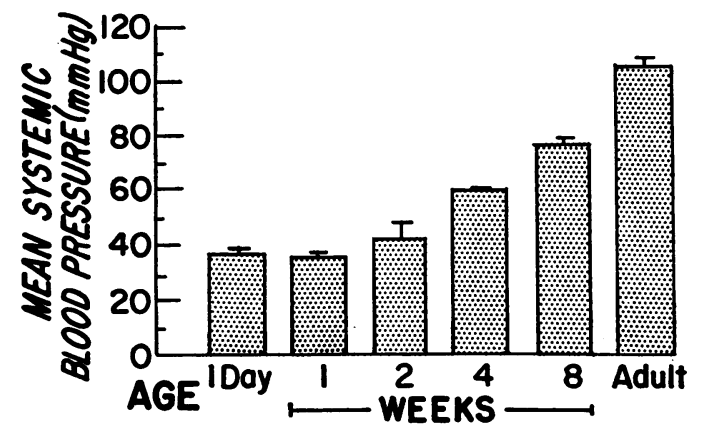

Fig. 2. Development of BLOOD PRESSURE IN THE DOG. Mean pressures were recorded before the start of each experiment. Each bar represents the mean of five animals. Lines indicate standard errors.

The duration of the angiotensin response is not significantly different from the adult by the time the dog is 1 week old. At 1 day these responses are significantly greater $(p<0.05)$ than those in the 1 -week-old to adult animals.

Systemic blood pressure. In these experiments we noted that the systemic blood pressure increased with the increasing age of the animal (Figure 1). The average values of the mean systemic blood pressures at the different ages (measured before the start of each experiment) are depicted in Figure 2. The blood pressures of the 1-day- and 1- and 2-week-old animals are approximately the same, whereas the pressures at 4 and 8 weeks are significantly larger than those at the previous three age groups $(\mathrm{p}<0.01,0.001$, respectively).

\section{Discussion}

Electrical stimulation of the lumbar sympathetic nerves innervating the vessels of the dog hindquarters produced two distinct types of responses that were dependent on the age of the animal. Nerve stimulation consistently produced vasodilatation in the 1-day- and 1- and 2-weekold animals, whereas vasoconstriction of increasing magnitude was seen in the 4- and 8-week-old and adult animals. Since the decrease in peripheral resistance was abolished by atropine, it is reasonable to assume that the neurotransmitter released upon stimulation of the sympathetic chain was acetylcholine. The presence of cholinergic fibers in the lumbar sympathetic chain of the dog is well documented (11). These fibers have their origin in the central nervous system $(12,13)$, but at present the functional significance of this sympathetic vasodilator pathway remains obscure. Our observations suggest that the cholinergic portion of the sympathetic nervous system is functional at an earlier time after birth than is the adrenergic component.

Since the vasoconstriction produced by nerve stimulation was significantly smaller in the 4and 8-week-old animals as compared to adults, it is reasonable to assume that the development of adrenergic vasomotor function begins between 2 and 4 weeks of age and progresses to functional maturity some time between 8 weeks and adulthood. The factors responsible for the absence of adrenergic function in the newborn have not been determined in this study. It is clear that biochemical studies are needed to determine if factors such as lack of catecholamine synthesizing enzymes or failure to bind or store norepinephrine or both contribute to the immaturity of the adrenergic system noted in the present study.

Under the conditions of the experiments mean systemic blood pressure of the 1-day-old puppy was $37 \mathrm{~mm} \mathrm{Hg}$, and it increased with age until it averaged 78 and $105 \mathrm{~mm} \mathrm{Hg}$ in the 8-week-old and adult animals, respectively. These findings agree with other reports noting the progressive increase of blood pressure in the newborn (14 18). Mott (19) has suggested that the rise in blood pressure is due to increasing activity of the autonomic nervous system with the establishment of sympathetic vasoconstrictor tone in the systemic circulation. We have observed that the onset of vasoconstriction induced by nerve stimulation coincides with the significant increase in systemic blood pressure (Figures 1 and 2) in the 4- and 8-week-old dogs. This evidence would thus seem to support the explanation suggested by Mott for this phenomenon. It should be emphasized, however, that the mechanisms responsible for the graded increase in blood pressure corresponding to age have not been determined. The relative contributions of changes in cardiac function, vascular reactivity and tone, and autonomic activity to the end result, which is a gradual increase in systemic blood pressure, require further investigation.

Epinephrine and norepinephrine in equal doses produced similar increases in perfusion pressure regardless of age. The average weight ( \pm stand- 
ard error) of each age group of animals was as follows : 1 day, $337 \pm 42 \mathrm{~g}$; 1 week, $423 \pm 42 \mathrm{~g}$; 2 weeks, $646 \pm 28 \mathrm{~g} ; 4$ weeks, $1.12 \pm 0.2 \mathrm{~kg} ; 8$ weeks, $2.2 \pm 0.2 \mathrm{~kg}$; and adults, $13.7 \pm 0.7 \mathrm{~kg}$. On a weight basis these age groups received the following average larger amounts of drug than the adult: 1 day, 39 times; 1 week, 31 times; 2 weeks, 20 times; 4 weeks, 12 times; and 8 weeks, 6 times. Since the catecholamines elicited essentially equivalent increments of perfusion pressure even though there was a wide difference in dose (on a weight basis) at the different ages, it is quite apparent that the younger animals were considerably less sensitive to the constrictor action of the catecholamines than the older animals. Diminished sensitivity of neonate vasculature was also noted with two other vasoconstrictors, angiotensin and tyramine. There are several possible explanations for the subsensitivity of vascular smooth muscle to constrictor agents in the newborn. Conceivably the number of "receptors" available for interaction with these materials may be limited, or the contractile protein within the muscle may be unable to develop tension in a manner comparable to that found in the adult animal. Unfortunately these and other explanations are purely speculative.

It is evident from the half-life data that the durations of action of epinephrine and norepinephrine are significantly longer in the newborn than the adult. After 4 weeks of age the duration of these responses is not different when compared to the mature animals. These observations suggest that mechanisms responsible for termination of catecholamines may be influenced by the functional state of the sympathetic adrenergic fiber. Brody has previously made similar observations in experiments on immunologically sympathectomized rats (20). These animals lack functional sympathetic nerves and demonstrate a significant decrease in their ability to terminate epinephrine and norepinephrine pressor responses in the hindquarters.

Although striking differences in vascular responses between newborn and adult dogs were noted in this study, several questions remain unanswered. All of the experiments were performed under barbiturate anesthesia. Even though every attempt was made to use an equivalent light plane of anesthesia at all ages, the pos- sibility must be entertained that the sympathetic nervous system or vascular smooth muscle of the young animals or both were exceptionally sensitive to the depressant effects of the anesthetic. Although differential sensitivity to anesthesia could have contributed to the present findings, it is unlikely that this factor could in toto explain the qualitatively different responses to nerve stimulation and great difference in reactivity to vasoconstrictors between adult and newborn dogs.

The problem of species variability must also be considered. This study demonstrates that the adrenergic sympathetic fibers that innervate the hindquarters of the dog are not functional for several weeks following birth. In view of the possibility that wide differences among species may exist with respect to maturity of the autonomic nervous and cardiovascular systems at birth, the present findings are applicable only to the canine species. Further studies of the type reported here will have to be carried out on a number of other species before a general concept of functional neuroeffector immaturity in the newborn can be established with certainty.

\section{Summary}

Electrical stimulation of the sympathetic nerves to the hindlimbs of the newborn dog produces a change in vascular resistance that is dependent on age. Stimulation consistently elicits vasodilatation until 2 weeks of age, and this dilatation is effectively abolished by atropine. Nerve stimulation in animals older than 2 weeks produces an increase in vascular resistance that becomes progressively greater in magnitude with age. These results indicate that the cholinergic portion of the sympathetic lumbar chain is functional at an earlier time following birth than the adrenergic component The observations confirm that systemic blood pressure increases with increasing maturity of the animal. The newborn is subsensitive to the constrictor action of epinephrine, norepinephrine, tyramine, and angiotensin, and the sensitivity increases with increasing age. Analysis of durations of pressor responses indicates that the younger animal is unable to terminate the action of catecholamines and angiotensin as rapidly as the adult. 


\section{References}

1. Clark, G. A. The development of blood pressure reflexes. J. Physiol. (Lond.) 1934, 83, 229.

2. Bauer, D. J. The effect of asphyxia upon the heart rate of rabbits at different ages. J. Physiol. (Lond.) 1938, 93, 90.

3. Hutchinson, E. A., C. J. Percival, and I. M. Young. Development of cardiovascular responses in the kitten. Quart J. exp. Physiol. 1962, 47, 201.

4. Downing, S. E. Baroreceptor reflexes in newborn rabbits. J. Physiol. (Lond.) 1960, 150, 201.

5. Dawes, G. S., J. J. Handler, and J. C. Mott. Some cardiovascular responses in foetal, new-born and adult rabbits. J. Physiol. (Lond.) 1957, 139, 123.

6. Dawes, G. S. Changes in the circulation at birth. Brit. med. Bull. 1961, 17, 148.

7. Cross, K. W., and J. L. Malcolm. Evidence of carotid body and sinus activity in newborn and foetal animals. J. Physiol. (Lond.) 1952, 118, $10 \mathrm{P}$.

8. Brody, M. J., R. A. Shaffer, and R. L. Dixon. A method for the study of peripheral vascular responses in the rat. J. appl. Physiol. 1963, 18, 645.

9. Beck, L. Active reflex dilatation in the innervated perfused hind leg of the dog. Amer. J. Physiol. 1961, 201, 123.

10. Snedecor, G. W. Statistical Methods, 5th ed. Ames, Iowa State College Press, 1956.

11. Bülbring, E., and J. H. Burn. The sympathetic dilator fiber in the muscles of the cat and dog. J. Physiol. (Lond.) 1935, 83, 483.
12. Eliasson, S., B. Folkow, P. Lindgren, and B. Uvnäs. Activation of sympathetic vasodilator nerves to the skeletal muscles in the cat by hypothalamic stimulation. Acta. physiol. scand. 1951, 23, 333.

13. Eliasson, S., P. Lindgren, and B. Uvnäs. Representation of the hypothalamus and the motor cortex in the dog of the sympathetic vasodilator outflow to the skeletal muscles. Acta. physiol. scand. 1952, 27, 18 .

14. Holland, W. W., and I. M. Young. Neonatal blood pressure in relation to maturity, mode of delivery, and condition at birth. Brit. med. J. 1956, 2, 1331.

15. Ashworth, A. M., and G. A. Neligan. Changes in the systolic blood-pressure of normal babies during the first twenty-four hours of life. Lancet 1959, 1, 804.

16. Woodbury, R. A., M. Robinow, and W. F. Hamilton. Blood pressure studies on infants. Amer. J. Physiol. 1938, 122, 472.

17. Forfar, J. O., and M. A. Kibel. Blood pressure in the newborn estimated by the flush method. Arch. Dis. Childh. 1956, 31, 126.

18. Moss, A. J., W. Liebling, and F. H. Adams. The flush method for determining blood pressure in infants. Pediatrics 1958, 21, 950.

19. Mott, J. C. The Stability of the Cardiovascular System. Somatic Stability in the Newly Born, A Ciba Foundation Symposium. G. E. W. Wolstenholme and M. O'Connor, Eds. Boston, Little, Brown, 1961.

20. Brody, M. J. Cardiovascular responses following immunological sympathectomy. Circulat. Res. $1964,15,161$ 